\title{
How, Where and When to Make a Biopsy?
}

\section{Burg G}

Professor \& Chairman Emerald, Department of Dermatology, University of Zuerich.

\author{
Address for correspondence \\ Guenter Burg, MD \\ Prof \& Chairm. emer. \\ Dermatology, University of Zuerich \\ CH 8124 Maur/ZH \\ Haldenstr. 14 \\ E-mail: g.burg@access.uzh.ch
}

\begin{abstract}
Considering the more than 2000 dermatoses it gets clear, that clinical morphology alone in many cases is not sufficient for making a correct diagnosis. Histomorphology can provide additional significant information in many but not in all cases. In order to save efforts, patient's inconvenience and costs it is important to know not only how and where to make a biopsy but also when a dermatopathologic investigation is mandatory and helpful in order to avoid misdiagnoses and wasting patients distress, time and money. Without being comprehensive some typical examples for various possible constellations are described.
\end{abstract}

Key words: dermatopathology, diagnosis, biopsy, clinic-histologic correlation

\section{Introduction}

$\mathrm{C}$ linical dermatology and dermatopathology are two sides of one coin. They mutually complement each other. In clinical dermatology one looks at the skin from outside, in dermatopathology one looks at the same thing from "inside". The microscope "translates" the clinical macromorphologic features into the corresponding histomorphologic terminology. In order to combine both to a meaningful diagnosis it is mandatory, that the clinician and the (dermato-) pathologist communicate and understand each other and discuss the clinico-pathologic correlations ${ }^{1,2}$. Treating patients for a wrong diagnosis may not only harm the patient but also generate unnecessary costs. Therefore, taking a biopsy should be considered whenever the clinical diagnosis is not clear. The three basic questions in this context are: how, where and when to make a biopsy.

\section{How to make a biopsy}

The excisional biopsy is the optimal procedure, since it not only provides a sample of the full lesion including surrounding normal skin but also is a therapeutic intervention by removing the pathologic lesion. Limitation may be given by the size of the lesion. Small single tumours are most appropriate for an excisional biopsy. The sample tissue should not be larger than 10 to $20 \mathrm{~mm}$ in order to guarantee proper fixation in an appropriate vessel, in which the sample is completely surrounded by buffered formalin.

An incisional biopsy takes only part of the lesion, which is too big to be completely excised. Like in the excisional biopsy, a spindle shaped tissue sample is excised usually from the border with the scalpel, cutting vertically to the skin surface.

The shave biopsy removes only the superficial part of a lesion, including the epidermis and the upper part of the dermis, leaving the lower dermal parts and the subcutis. This is diagnostically sufficient, when the pathologic changes are in the upper skin levels, like for example in nodular prurigo.

The punch biopsy is easy to perform with disposable devices. Depending of the size of the punch, wound closure may be neglected.

When superficial material is sufficient for microscopic investigation, smear or debris is sampled. Processing of debris material is more difficult than with a solid biopsy sample due to the small pieces floating in the fixation medium. 


\section{Where to make a biopsy?}

Basically there are five options to take a biopsy.

In tumorous lesions, which are too big for being totally excised with an excisional biopsy, the sample will be taken from the centre of the lesion either by spindle excision or by a punch biopsy. In order to provide optimal material for the processing of the tissue sample (fixation, cutting, microscopic investigation) it is important that the tissue is not necrotic.

Taking a (spindle) biopsy from the border of the lesion, including normal and diseased parts of the skin, is indicated in inflammatory dermatoses, especially if the lesion shows central regressing like for example in superficial fungus infection, psoriasis or in granuloma annulare.

In deep inflammatory conditions with involvement of the lower parts of the dermis and the subcutaneous fat tissue, like in erythema nodosum or panniculitis, a deep biopsy including the subcutis is indicated. When making a punch biopsy, the size of the punch should be as wide as possible.

Superficial shave biopsy is a suboptimal procedure. The advantage is, that no wound closure is needed. Usually it is sufficient in dermatoses, whose major pathologic changes are located in the epidermis and the upper dermis like in psoriasis, eczema, nodular prurigo, lichen planus.

Scarification and sampling debris is an appropriate procedure in for example leishmaniasis or lepromatous leprosy. The debris collected may be difficult to be kept together in the embedding procedure. Anatomical structures are certainly lost with this material. Contamination with blood should be avoided.

\section{When to make a biopsy?}

The gold standard for making a diagnosis is the clinical evaluation, based on localisation of the lesion(s) and their distribution and the morphology of the single primary lesion. This also has been the basis for the classification of dermatoses since the work of Willan and Bateman 200 years ago ${ }^{3}$, which in general still is followed to day in most textbooks. In $70-80 \%$ of the most common dermatoses in rural areas ${ }^{4,5}$ (eczemas, acne, pigment disorders, urticaria) proper diagnosis can be made by clinical evaluation without any additional diagnostic procedure. However the remaining 20-30\% include rare diseases or dermatoses simulating common dermatoses, which may lead to misdiagnosis and mistreatment. This may be harmful for the patient and generates loss of time and unnecessary costs.

In cases with doubtful clinical signs, a biopsy is mandatory, when the histologic features can be clearly diagnostic and provide a high diagnostic impact. Examples are toxic epidermal necrolysis (TEN) versus staphylococcal scaled skin syndrome (SSSS); scalp psoriasis versus seborrheic dermatitis of the scalp; plaque psoriasis of the trunk versus pityriasis rubra pilaris or patch/plaque type mycosis fungoides; acute eczema versus pemphigus foliacius; psoriatic erythroderma versus sézary syndrome; urticaria or urticaria vasculitis versus acute febrile neutrophilic dermatosis (sweet syndrome); pityriasis rosea versus secondary syphilis; lupus erythematosus versus rosacea; lichen planus of the anogenital region versus lichen sclerosus et atrophicus; intertriginous eczema or psoriasis versus langerhans cell histiocytosis (histiocytosis X); paget's disease or perimammillar psoriasis versus eczema; melanoma versus junctional nevus or angiokeratoma. There are many other dermatoses which clinically may look alike and can only be differentiated by appropriate histologic investigation.

Special types of basal cell carcinomas tend to grow subclinically beyond visible borders. In these cases Microscopic controlled surgery (Mohs technique) ${ }^{6}$ is indicated in order to remove all branches of the tumor, which in case of uncomplete excision give rise to recurrences. Especially in facial localisation close to eyes, nose, ear or mouth repeated excisions may result in cosmetic misconfiguration. By complete 3-dimensional evaluation of the excised tissue sample(s) with appropriate labelling of the borders according to the Mohs technique, the tumorous lesion can be completely removed by minimal sacrifice of normal tissue.

This technique is extremely helpful but very elaborate and time consuming and therefore should be restricted to very special cases with a high threat of recurrence due to incomplete excision.

Making a biopsy in dermatoses, which clinically present with a clear-cut diagnosis, is optional and can be performed just for having additional confirmation. It creates additional costs without providing appropriate benefit, except when done for research purposes.

In some dermatoses, microscopic investigation can be even confusing, since the histologic features may lead to wrong diagnostic conclusions. In these cases 
the clinical features are pivotal for the diagnosis, since the histology may reveal similar or even identical pictures of different diseases. Examples among inflammatory dermatoses are transient acantholytic dermatosis (Grover's disease) which shows similar acantholytic split formation as seen in pemphigus benignus familiaris (Hailey-Hailey).However the clinical presentations with small papules in Grover's disease and large plaques mainly in the large skin folds are completely different. Chronic graft versus host (GVH)-reaction histologically simulates lichen planus; the history however certainly differentiates both conditions. The histologic changes in diffuse scleroderma are identical with those seen in localised scleroderma (Morphea), whereas the clinical features are different. It may be impossible to differentiate histologically the changes seen in dermatomyositis from acute systemic lupus erythematosus. The clinical symptoms elucidate the correct diagnosis. With reference to tumorous conditions, the histologic differentiation of keratoacanthoma (KA) from well differentiated squamous cell carcinoma (SCC) is based on history, which is in the range of weeks in KA and months in SCC: Anaplastic large cell lymphoma, CD 30 positive and lymphomatoid papulosis show similar histologic but different clinical fatures and courses.

There are also situations, in which neither the clinical nor the histologic presentation are sufficient for making a correct diagnosis. For example pseudolymphomatous infiltrate and cutaneous follicle center cell lymphoma clinically and histologically look perfectly alike and it is the course-spontaneous regression or continuously progressive- which finally will reveal the correct diagnosis.

Many other diseases can create diagnostic problems. The list is far from being comprehensive, but some more dermatoses shall be mentioned: urticaria versus urticaria-vasculitis, parapsoriasis versus initial mycosis fungoides or nonspecific eczema, spindle cell tumors of various histogenetic especially mesenchymal origin, nevus Spitz versus spitzoid melanoma and many others.

Nevertheless looking not only for the clinical feature but also at the histology is the clue in many diagnostic dilemmas and can avoid mistreatment, which is harmful not only for the patient but also for the public healthcare budget.

The Nepalese Society for Dermatopathology has been founded 3 years ago under the patronage of the DISHARC-clinic in Kathmandu. Now there is an increasing number of Dermatopathologists in Nepal, who are trained in both, clinical dermatology and dermatopathology. They are prepared for assisting with their expertise in providing appropriate consultations for better healthcare for patients with ambiguous dermatologic diagnoses.

\section{References}

1. Burg, G., W. Kempf, Kutzner H, Feit J, Laszlo Karai L, et al. (2015). Atlas of Dermatopathology: Practical Differential Diagnosis by Clinicopathologic Pattern. Oxford, Wiley. https://doi.org/10.1002/9781118658307

2. Feit J, Kempf W, Jedlicková H, Burg G. Hypertext atlas of dermatopathology with expert system for epithelial tumors of the skin. J Cutan Pathol 2005 ;32(6): 433-7.

3. Willan R. and Bateman T. A practical Synopsis of Cutaneous Diseases. According to the arrangement of Dr. Willan, exhibiting a concise view of the diagnostic symptoms and the method of treatment. London: 1817.
4. Shrestha D P, Gurung D. Prevalence of skin diseases and impact on quality of life in hilly region of Nepal. Journal of Institute of Medicine 2012:34(3): 44-49. http://dx.doi.org/10.3126/ jiom.v34i3.8917.

5. Shrestha DP, Baral S. Frequency and pattern of Skin Disorders in Adolescents in a School of Kathmandu:.Journal of Institute of Medicine 2015; 37(1): 21-25.

6. Mohs, F. E. Mohs micrographic surgery. A historical perspective. Dermatol Clin 1989; 7(4): 609-611. 